\title{
An Integrated Signal and Power Integrity Analysis for Signal Traces Through the Parallel Planes Using Hybrid Finite-Element and Finite-Difference Time-Domain Techniques
}

\author{
Wei-Da Guo, Guang-Hwa Shiue, Chien-Min Lin, Member, IEEE, and Ruey-Beei Wu, Senior Member, IEEE
}

\begin{abstract}
This paper presents a numerical approach that combines the finite-element time-domain (FETD) method and the finite-difference time-domain (FDTD) method to model and analyze the two-dimensional electromagnetic problem concerned in the simultaneous switching noise (SSN) induced by adjacent signal traces through the coupled-via parallel-plate structures. Applying FETD for the region having the source excitation inside and FDTD for the remaining regions preserves the advantages of both FETD flexibility and FDTD efficiency. By further including the transmission-line simulation, the signal integrity and power integrity issues can be resolved at the same time. Furthermore, the numerical results demonstrate which kind of signal allocation between the planes can achieve the best noise cancellation. Finally, a comparison with the measurement data validates the proposed hybrid techniques.
\end{abstract}

Index Terms-Differential signaling, finite-element and finitedifference time-domain (FETD/FDTD) methods, power integrity (PI), signal integrity (SI), simultaneous switching noise (SSN), transient analysis.

\section{INTRODUCTION}

$\mathbf{I}$ N RECENT years, considerable attention has been devoted to time-domain numerical techniques to analyze the transient responses of electromagnetic problems. The finite-difference time-domain (FDTD) method proposed by Yee in 1966 [1] has become the most well-known technique because it provides a lot of attractive advantages: direct and explicit timemarching scheme, high numerical accuracy with a second-order discretization error, stability condition, easy programming, and minimum computational complexity [2]. However, it is often inefficient and/or inaccurate to use only the FDTD method to deal

Manuscript received March 3, 2006; revised November 6, 2006. This work was supported in part by the National Science Council, Republic of China, under Grant NSC 91-2213-E-002-109, by the Ministry of Education under Grant 93B40053, and by Taiwan Semiconductor Manufacturing Company under Grant 93-FS-B072.

W.-D. Guo, G.-H. Shiue, and R.-B. Wu are with the Department of Electrical Engineering and Graduate Institute of Communication Engineering, National Taiwan University, 10617 Taipei, Taiwan, R.O.C. (e-mail: f92942062@ntu.edu.tw; d90942002@ntu.edu.tw; rbwu@ew.ee.ntu.edu.tw).

C.-M. Lin is with the Packaging Core Competence Department, Advanced Assembly Division, Taiwan Semiconductor Manufacturing Company, Ltd., 30077 Taiwan, R.O.C. (e-mail: chienmin_lin@tsmc.com).

Color versions of one or more of the figures in this paper are available online at http://ieeexplore.ieee.org.

Digital Object Identifier 10.1109/TADVP.2007.901595 with some specific structures. Hybrid techniques, which combine the desirable features of the FDTD and other numerical schemes, are therefore being developed to improve the simulation capability in solving many realistic problems.

First, the $\operatorname{FDTD}(2,4)$ method with a second-order accuracy in time and a fourth-order accuracy in space was incorporated to tackle the subgridding scheme [3] and a modified form was employed to characterize the electrically large structures with extremely low-phase error [4]. Second, the integration with the time-domain method of moments was performed to analyze the complex geometries comprising the arbitrary thin-wire and inhomogeneous dielectric structures [5], [6]. Third, the flexible finite-element time-domain (FETD) method was introduced locally for the simulation of structures with curved surfaces [6]-[8].

With the advent of high-speed digital era, the simultaneous switching noise (SSN) on the dc power bus in the multilayer printed circuit boards (PCBs) causes paramount concern in the signal integrity and power integrity (SI/PI) along with the electromagnetic interference (EMI). One potential excitation mechanism of this high-frequency noise is from the signal traces which change layers through the via transition [9]-[11]. In the past, the transmission-line theory and the two-dimensional (2-D) FDTD method were combined successfully to deal with the parallel-plate structures having single-ended via transition [12], [13]. Recently, the differential signaling has become a common wiring approach for high-speed digital system designs in benefit of the higher noise immunity and EMI reduction. Nevertheless, for the real layout constraints, the common-mode currents may be generated from various imbalances in the circuits, such as the driver-phase skew, termination diversity, signal-path asymmetries, etc. Both the differentialand common-mode currents can influence the dc power bus, resulting in the SSN propagating within the planes.

While applying the traditional method to manage this case, it will need a much finer FDTD mesh to accurately distinguish the close signals transitioning through the planes. Such action not only causes the unnecessary waste of computer memory but also takes more simulation time. In order to improve the computational efficiency, this paper incorporates the FETD method to the small region with two or more signal transitions inside, while the other regions still remain with the coarser FDTD grids. While the telegrapher's equations of coupled transmission lines are further introduced to the hybrid FETD/FDTD techniques, the 


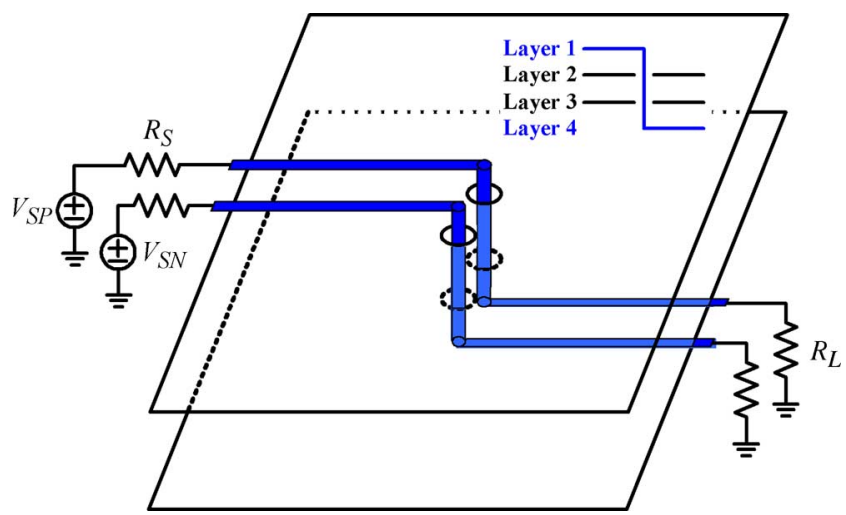

Fig. 1. A typical four-layer differential-via structure.

SI/PI co-analysis for differential traces through the planes can be accomplished as demonstrated in Section II and the numerical results are shown in Section III. For a group of signal vias, the proposed techniques can also tell which kind of signal allocation to achieve the best performance as presented in Section III. Section IV thus correlates the measurement results and their comparisons, followed by brief conclusions in Section V.

\section{Simulation Methodology}

A typical differential-via structure in a four-layer board is illustrated in Fig. 1. Along the signal-flow path, the whole structure is divided into three parts: the coupled traces, the coupled-via discontinuities, and the parallel plates. This section will present how the hybrid techniques integrate the three parts to proceed with the SI/PI co-simulation. At last, the stability consideration and computational complexity of the hybrid techniques are discussed as well.

\section{A. Circuit Solver}

With reference to Fig. 2, if the even/odd mode propagation coefficients and characteristic impedances are given, it is recognized that the coupled traces can be modeled by the equivalent $L C$ ladder circuits, and the lossy effects can be well approximated with the average values of individual $R_{\mathrm{ac}}$ and $G_{\mathrm{ac}}$ over the frequency range of interest. The transient signal propagation is thus characterized by the telegrapher's equations with the central-difference discretization both in time and space domains. The approach to predict the signal propagation through the coupled-via discontinuities is similar to that through the coupled traces except for the difference of model-extracting method.

To characterize the coupled-via discontinuities as depicted in Fig. 1, the structure can be separated into three segments: the via between the two solid planes, and the via above (and under) the upper (and lower) plane. Since the time delay of signals through each segment is much less than the rising edge of signal, the coupled-via structure can be transformed into a SPICE passive network sketched in Fig. 3 by full-wave simulation [14], where $V_{p}$ represents the voltage of SSN induced by the current $I_{v}$ on Ls2. By linking the extracted circuit models of coupled-via discontinuities, both the top- and bottom-layer traces together with suitable driving sources and load terminations, the transient waveforms throughout the interconnects are then characterized and can be used for the SI analyses.

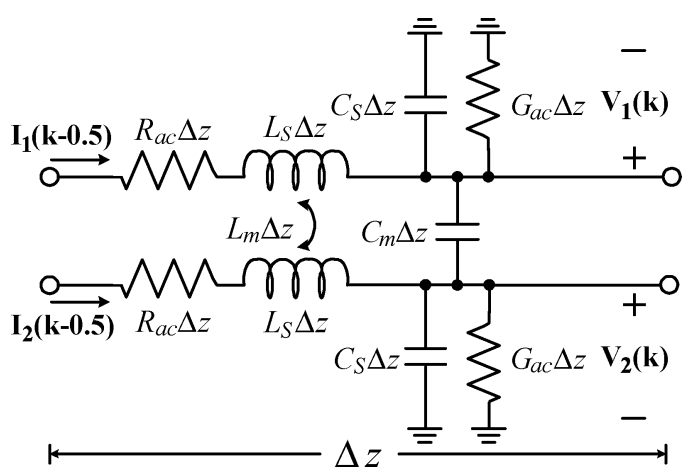

Fig. 2. The $k$ th element of equivalent circuit model of coupled transmission lines.

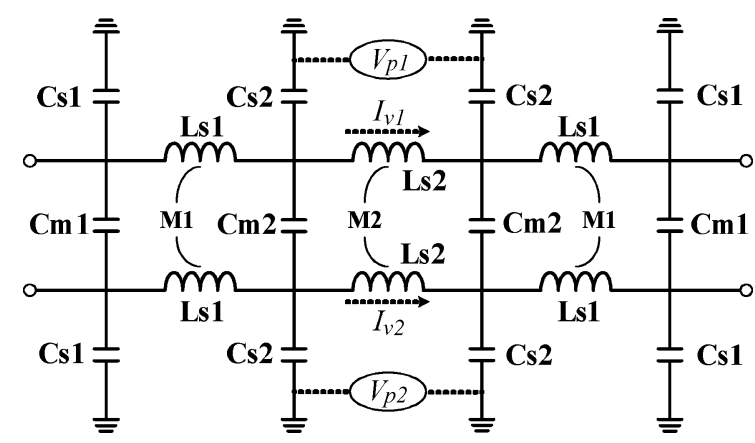

Fig. 3. Equivalent circuit model of coupled-via structures.

\section{B. Plane Solver}

As for the parallel-plate structure, because the separation between two solid planes is much smaller than the equivalent wavelength of signals, the electromagnetic field inside is supposed to be uniform along the vertical direction. Thence, the 2-D numerical technique can be applied to characterize the SSN effects while the FETD method is set for the small region covering the signal transitions and the FDTD scheme is constructed in the most regular regions.

The FETD algorithm [15] starts from Maxwell's two curlequations and the vector equation is obtained by

$$
\nabla \times \nabla \times \vec{E}+\mu \varepsilon \frac{\partial^{2} \vec{E}}{\partial t^{2}}+\mu \frac{\partial \vec{J}}{\partial t}=0 \quad \text { in } \Omega
$$

where $\vec{E}$ and $\vec{J}$ denote the electric field and current density, respectively, in the lossless volume $\Omega$. Applying the weak-form formulation or the Galerkin's procedure to (1) gives

$$
\begin{aligned}
& \int_{\Omega}(\nabla \times \vec{W} \cdot \nabla \times \vec{E}) d \Omega+\mu \varepsilon \int_{\Omega} \vec{W} \cdot \frac{\partial^{2} \vec{E}}{\partial t^{2}} d \Omega \\
& \quad+\frac{\mu_{r}}{c} \oint_{\Gamma_{\infty}} \vec{W} \cdot\left(\frac{\partial \vec{E}}{\partial t} \times \hat{n}\right) d \Gamma+\mu \int_{\Omega} \vec{W} \cdot \frac{\partial \vec{J}}{\partial t} d \Omega=0
\end{aligned}
$$

where $\vec{W}$ is the weighting function that can be arbitrarily defined. In use of the finite-element method, the variational formula is thus discretized to implement the later numerical computation. In the present case, the linear basis function is chosen to express the fields inside each triangular element. After taking the volume integration over each element and assembling the 


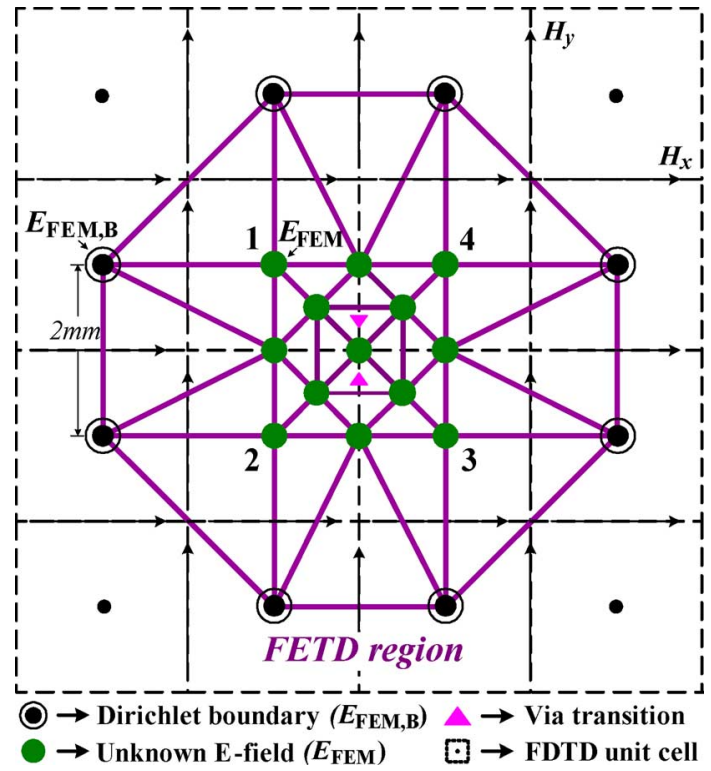

Fig. 4. FEM mesh in the source region and its interface with the FDTD grids.

integrals from all the elements, (2) can be simplified into a matrix form of

$$
[C] \frac{\partial^{2} E}{\partial t^{2}}+[D] E+[F] \frac{\partial J}{\partial t}=0
$$

where $E$ and $J$ are the coefficient vectors of electric field and current density, respectively. In addition, the values of all matrix elements in (3) are formulated as

$$
[C]_{i j}=\mu \varepsilon \int_{\Omega} \vec{W}_{i} \cdot \vec{W}_{j} d \Omega, \quad[F]_{i}=\mu \int_{\Omega} \vec{W}_{i} d \Omega
$$

and

$$
[D]_{i j}=\int_{\Omega}\left(\nabla \times \vec{W}_{i} \cdot \nabla \times \vec{W}_{j}\right) d \Omega .
$$

For the mesh profile as illustrated in Fig. 4, the FETD region is chosen to be a block replacing the prime FDTD region into which the via transition penetrates. This is an initial value problem in time with the previous $E_{\mathrm{FEM}}$ and $\partial J / \partial t$ being the initial conditions as well as the boundary value problem in space with $E_{\mathrm{FEM}, \mathrm{B}}^{n+1}$ being Dirichlet boundary condition. To solve the initial value problem in (3), the time derivative of electric field is approximated by the central difference, that is

$$
\left.\frac{\partial^{2}}{\partial t^{2}} E\right|_{t=n \Delta t} \approx \frac{1}{(\Delta t)^{2}}\left(E^{n+1}-2 E^{n}+E^{n-1}\right) .
$$

As for the electric field in the second term of (3), it can be formulated by the Newmark-Beta scheme [16] to be read as

$$
\left.E\right|_{t=n \Delta t} \approx \frac{1}{4}\left(E^{n+1}+2 E^{n}+E^{n-1}\right) .
$$

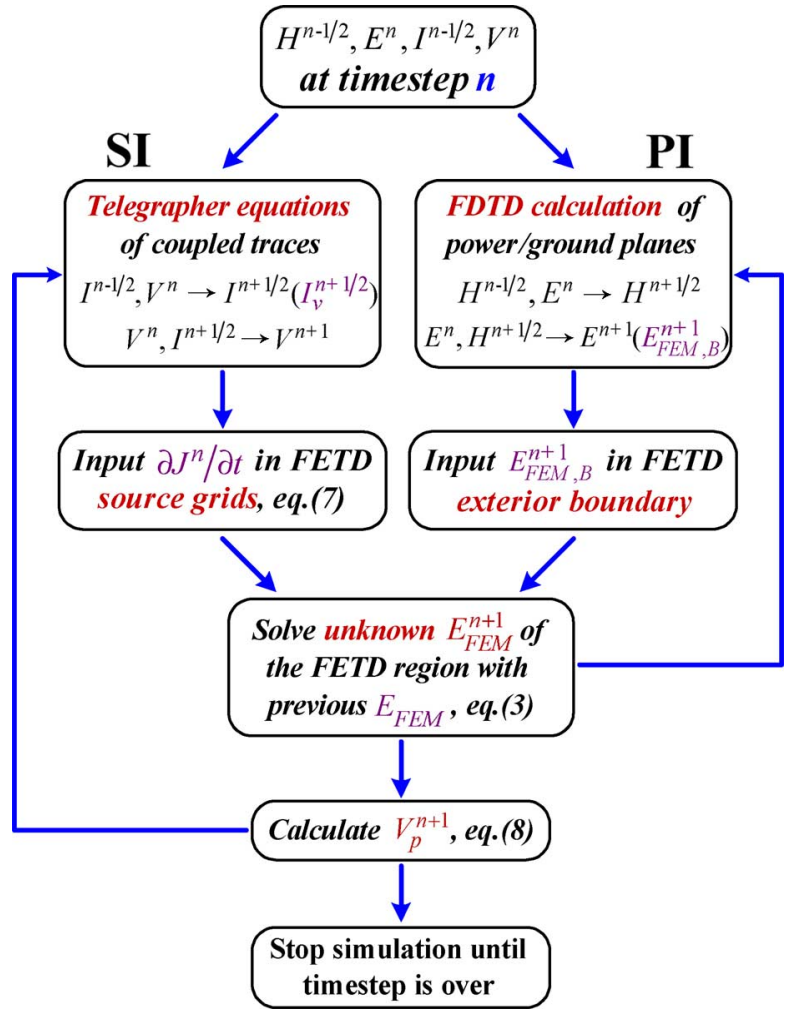

Fig. 5. Simulation flowchart of hybrid FETD and FDTD techniques to perform the SI/PI co-analysis for the coupled-via structure as illustrated in Fig. 1.

Moreover, in the triangular elements with the via transition inside, the $\partial J / \partial t$ term in (3) as expressed by

$$
\frac{\partial J^{n}}{\partial t} \approx \frac{1}{\Delta t}\left(\frac{I_{v}^{n+0.5}-I_{v}^{n-0.5}}{\text { grid area }}\right)
$$

is needed to serve as the excitation of the parallel-plate structure with the current $I_{v}$ shown in Fig. 3 through the via structure between Layers 2 and 3. It is worth noting that the via transition should be placed on the bary-center of each triangular element to achieve better accuracy.

The hand-over scheme for the field in the overlapped region of FDTD and FETD can be depicted in Fig. 5. Given the boundary field $E_{\mathrm{FEM}, \mathrm{B}}^{n+1}$ calculated by the FDTD algorithm at the time step $n+1$, all the field $E_{\mathrm{FEM}}^{n+1}$ in the FETD region can be acquired through the matrix solution of (3). The SSN voltage $V_{p}$ in Fig. 3 is then determined by

$$
V_{p}^{n+1}=E_{\text {average }}^{n+1} \cdot d
$$

where $E_{\text {average }}^{n+1}$ is the averaging value of nodal electric-fields enclosing the via transition, and $d$ is the separation between the planes. Once $V_{p}^{n+1}$ and $E_{\mathrm{FEM}}^{n+1}$ at the FETD mesh nodes (node 1, 2, 3, and 4 in Fig. 4) become available, together with the obtained voltage/current values from the circuit solver and electric/ magnetic fields of the FDTD region, the hybrid time-marching scheme for the next time step can be implemented and so on.

As a result of using the integrated schemes, the current $I_{v}$, arisen from the input signal through the via structure, can have the ability to induce the voltage noise propagating within the 


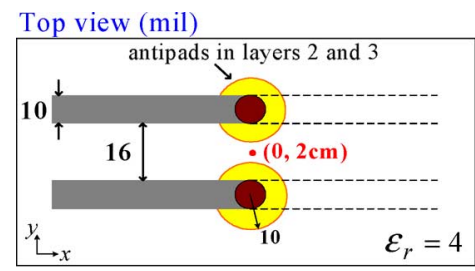

(a)

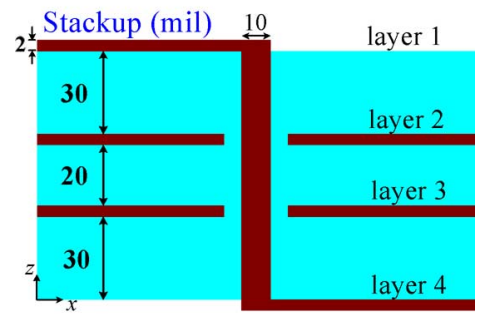

(b)

Fig. 6. Physical dimensions of coupled traces and via pair. (a) Top view $($ Unit $=$ mil $)$. (b) Side view

parallel plates. After a period of time, owing to the plane resonance and return path, the induced noise will cause the unwanted voltage fluctuation on the coupled traces by the presence of the finite SSN voltage $V_{p}$.

\section{Stability Problem and Computational Complexity}

It is not difficult to manifest that the FETD algorithm is unconditionally stable. Substituting (5), (6), and (7) into (3) yields the following difference equation:

$$
E^{n+1}=\left(2-[X]^{-1}[\mathrm{M}]\right) E^{n}-E^{n-1}-\xi^{n}[F]
$$

where

$$
[M]=\Delta t^{2}[D], \quad[X]=[C]+0.25 \Delta t^{2}[D]
$$

the superscript " -1 " denotes the matrix inverse and the factor

$$
\xi^{n}=\Delta t\left(\frac{I_{v}^{n+0.5}-I_{v}^{n-0.5}}{\text { grid area }}\right) .
$$

Without loss of generality, the time-stepping scheme in (9) is restated as

$$
E^{n}=\left(2-[X]^{-1}[\mathrm{M}]\right) E^{n-1}-E^{n-2}-\xi^{n-1}[F] .
$$

Applying the $Z$-transform technique to (11) and solving for $\widetilde{E}(z)$, defined as the $Z$-transform of $E^{n}$, the result reads

$$
\widetilde{E}(z)=\frac{-z \widetilde{\xi}(z)[F]}{z^{2}-\left(2-[X]^{-1}[M]\right) z+1}
$$

along with the dependent $\widetilde{\xi}(z)$, defined as the $Z$-transform of $\xi^{n-1}$ in (11). Regardless of the time step $\Delta t$, it can be easily deduced that the poles of (12) is just on the unit circle of $z$ plane. This proves that the time marching by (9) is absolutely stable. The stability condition of these hybrid techniques is thus governed by the transmission-line theory and the FDTD algorithm in the regular region, which are already known.

Concerning the computational complexity, because of the consistence of simulation engines used for the circuit solver,

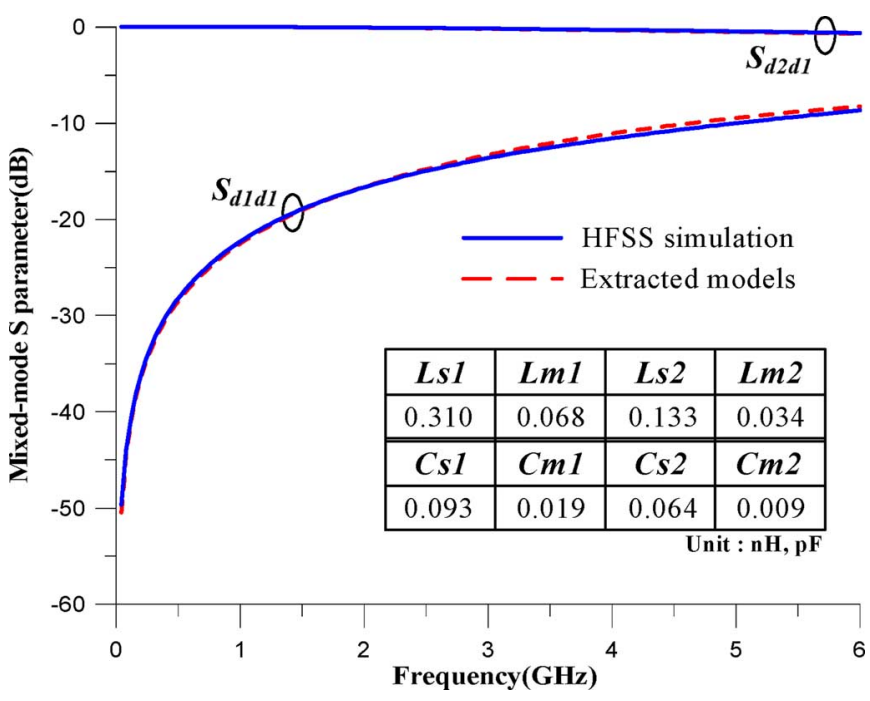

Fig. 7. Comparison of differential-mode $S$-parameters from HFSS simulation and the equivalent circuit as depicted in Fig. 3.

the only work is to compare the efficiency of the hybrid FETD/FDTD technique with that of the traditional FDTD method. In use of only the FDTD scheme for cell discretization, the grid size should be chosen at most the spacing between the adjacent via transitions. However, as depicted in Fig. 4, the hybrid techniques adopting the FEM mesh for the source region exhibit the great talent to segment the whole plane with the coarser FDTD grids. Owing to the sparsity of the FETD matrices in (4) and the much smaller number of unknowns, the computational time needed for each FETD operation can be negligible. The complexity of the hybrid techniques is therefore dominated by the FDTD divisions in the regular region. It is evident that the total simulation time of the 2-D FDTD algorithm is $O\left(N^{1.5}\right)$, where $N$ denotes the number of the division in the whole space [7]. The coarser the FDTD grids, the smaller the number of the grids and unknowns. Hence, the present hybrid techniques can preserve high accuracy without sacrificing the computational efficiency.

\section{NUMERICAL RESULTS}

\section{A. Coupled via Transition}

Consider the geometry in Fig. 1 but with the coupled-via structure being $2 \mathrm{~cm}$ away from the center of parallel plates, which is set as the origin of the $x-y$ plane. The size of the plane is $10 \times 10 \mathrm{~cm}^{2}$ and the separation between the two metal planes is 20 mils $(\approx 0.05 \mathrm{~cm})$. The physical dimensions of the coupled traces and via pair are depicted in Fig. 6. After extracting the $S$-parameters from the full-wave simulation, their equivalent circuit models of coupled-via structures as sketched in Fig. 3 can be thus constructed. In Fig. 7, it is found that the differential-mode $S$-parameters of equivalent circuit models are in good agreement with those from the HFSS simulations [14] and the extracted parasitic values of inductive and capacitive lumped-elements are also listed in the attached table.

The top-layer coupled traces are driven by differential Gaussian pulses with the rise time of 100 ps and voltage amplitude of $\pm 2 \mathrm{~V}$ while the traces are terminated with the matched 


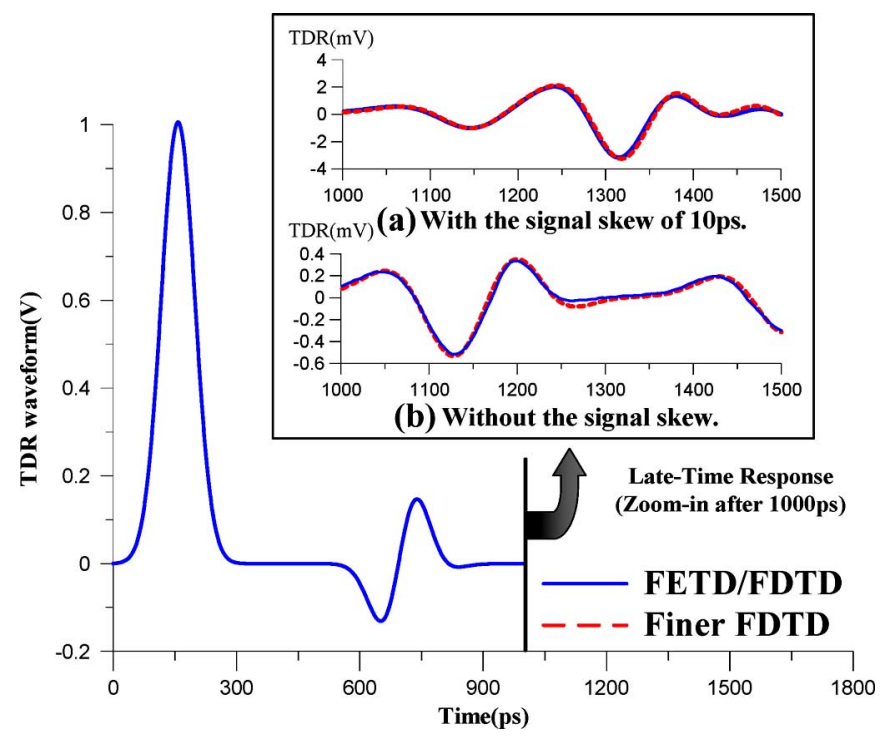

Fig. 8. Simulated TDR waveforms on the positive-signaling trace. (a) Latetime response for the signal skew of $10 \mathrm{ps}$ excluding the multireflection phenomenon of common-mode signal. (b) Late-time response while no signal skew.

TABLE I

COMPARISON OF COMPUTATIONAL COMPLEXITY BETWEEN THE TWO METHODS $($ TIME DURATION $=2.5 \mathrm{~ns})$

\begin{tabular}{|c|c|c|}
\hline & $\begin{array}{c}\text { Hybrid } \\
\text { technique }\end{array}$ & Finer FDTD \\
\hline Division size & $\begin{array}{c}\mathrm{ds}=2 \mathrm{~mm} ; \\
\mathrm{dt}=5 \mathrm{ps}\end{array}$ & $\begin{array}{c}\mathrm{ds}=0.66 \mathrm{~mm} \\
\mathrm{dt}=2.5 \mathrm{ps}\end{array}$ \\
\hline $\begin{array}{c}\text { The number of } \\
\text { division }(\boldsymbol{N})\end{array}$ & $50 \times 50$ & $150 \times 150$ \\
\hline $\begin{array}{c}\text { Simulation time } \\
\left(\mu N^{*} \text { timestep }\right)\end{array}$ & $0.8 \mathrm{sec}$ & $13.5 \mathrm{sec}$ \\
\hline
\end{tabular}

(CPU: Intel P4 3.0 GHz, RAM: 2 GHz)

loads at their ends. For simplicity, the transmission-line losses are not considered in the following analyses for the transient responses. By using the same mesh discretization as illustrated in Fig. 4, the resultant segmentation for the plane confines the flexible FEM mesh in the vicinity of via transitions and the coarser FDTD division with the size of $2 \times 2 \mathrm{~mm}^{2}$ elsewhere. Employing the perfect magnetic conductors for boundary conditions of the parallel-plate structure, the simulated TDR waveforms with and without the signal skew on the positive-signaling trace are presented in Fig. 8. In comparison of hybrid FETD/FDTD techniques and finer FDTD method with center-to-center via spacing $(\approx 0.66 \mathrm{~mm})$ as the grid size, the simulation results are in good agreement. Note that the voltage fluctuation before $900 \mathrm{ps}$ is induced by the incident signal passing through the coupled-via structure while the occurrence of late-time response is accompanied by the parallel-plate resonances. As for the signal skew of $10 \mathrm{ps}$, the voltage level of late-time response is found to be greater than that of no signal skew because of the existence of common-mode currents produced by the timing skew of differential signals.

Moreover, the simulation time of both methods should be proportional to the number of grids multiplied by the total time steps. As the physical time duration is fixed, the decrease of the FDTD division size would correspond to the increase of the total

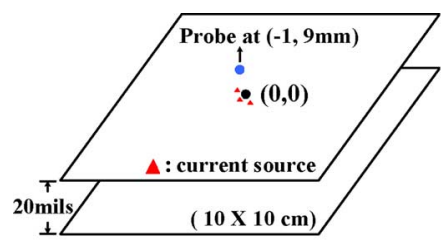

(a)

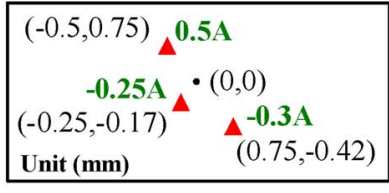

(b)

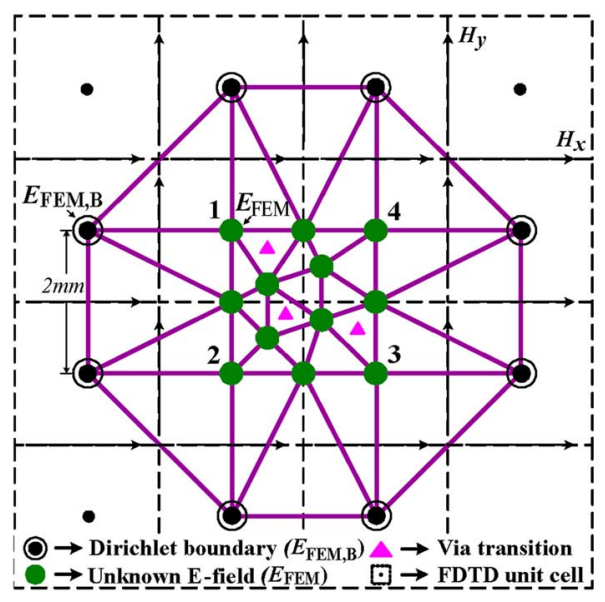

(c)

Fig. 9. Parallel plane with three current sources inside. (a) 3-D view. (b) Zoom-in view of three sources on the plane in (a). (c). FETD/FDTD mesh discretization.

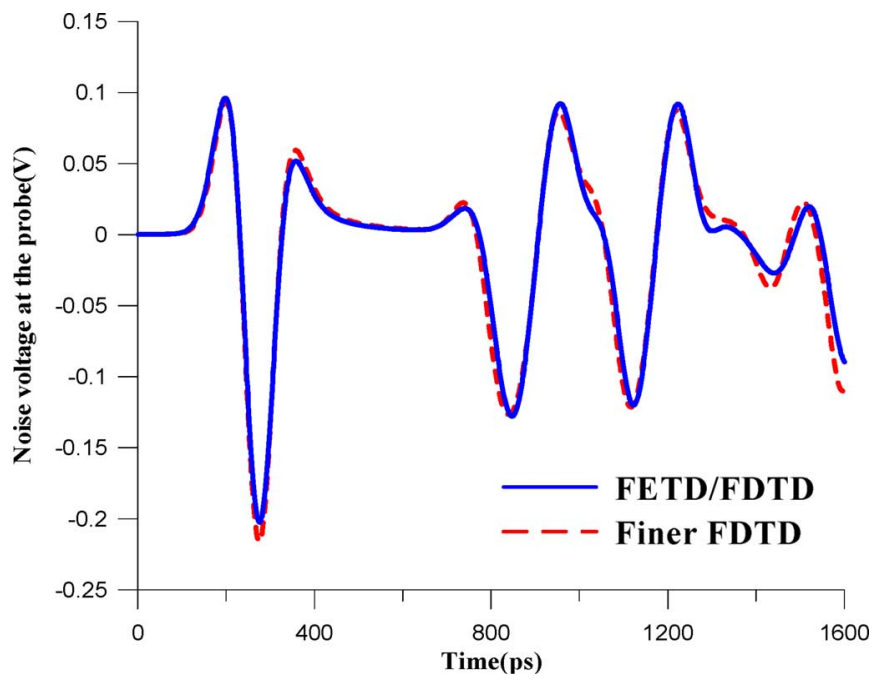

Fig. 10. Simulated noise waveforms at the preallocated probe in reference to Fig. 9(a).

time steps. Consequently, as shown in Table I, it is demonstrated that the computational efficiency of the hybrid techniques is indeed much better than that of the finer FDTD method.

\section{B. Multiple Source Transition}

In addition to a pair of differential-via structure, there can be a group of signaling vias distributed in the various regions of planes. Considering the parallel-plate structure in Fig. 9(a), three current sources are distributed around the center $(0,0)$ and a probe is located at $(-1 \mathrm{~mm}, 9 \mathrm{~mm})$ to detect the voltage noise induced on the planes. The FEM meshes for the source region and the interface with the FDTD region are shown in 


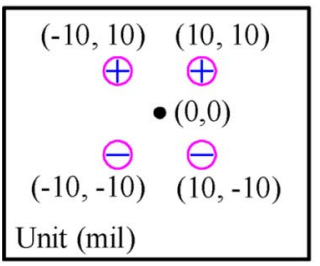

(a)

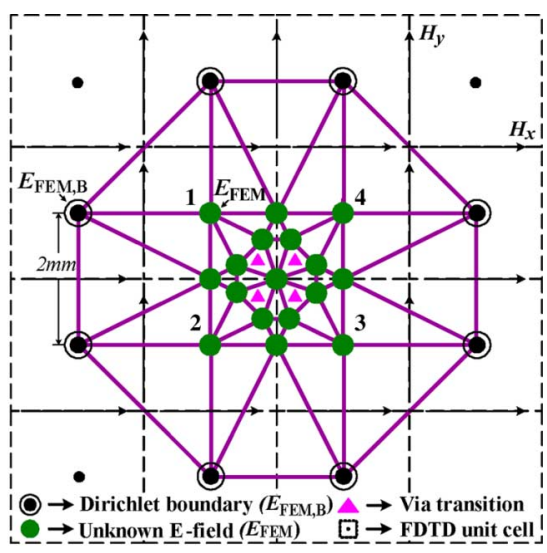

(b)
Fig. 11. Parallel-plate structure with two differential pairs of current sources inside in reference to Fig. 9(a). (a) Two differential pairs of sources on the plane in Fig. 9(a). (b) FETD/FDTD mesh discretization.

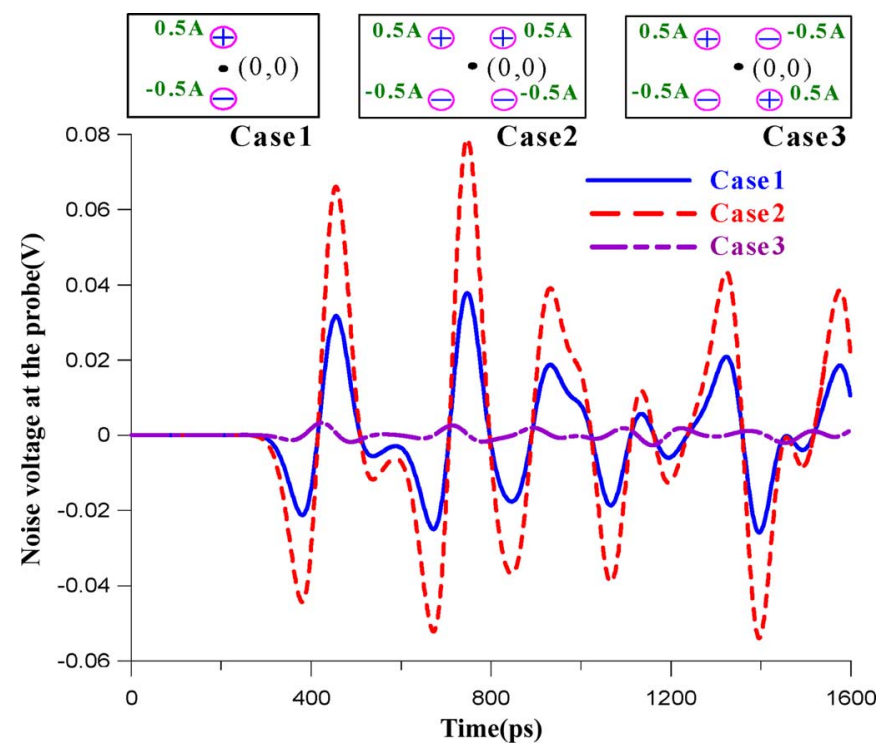

Fig. 12. Comparison of the simulated noise waveforms between three cases of differential-sources on the plane as in Fig. 9(a).

Fig. 9(c). The current sources are Gaussian pulses with the rise time of 100 ps and different current amplitudes of $0.5,-0.25$, and $-0.3 \mathrm{~A}$. With the same settings of boundary conditions, the simulated voltage noise waveforms at the preallocated probe referred to Fig. 9(a) are presented in Fig. 10. It is indicated that the hybrid FETD/FDTD techniques still reserves the great accuracy in predicting the traveling-wave behavior of plane noise.

In the modern digital systems, many high-speed devices employ the multiple differential-traces for the purpose of data transmission. These traces are usually close to each other and may simultaneously penetrate the multilayered planes through via transitions. Hence, it is imperious for engineers to know how to realize the best power integrity by suitably arranging the positions of differential vias.

Reconsidering the parallel plates in Fig. 9(a), instead, two differential-current sources around the center and the probe is relocated at $(-25 \mathrm{~mm},-25 \mathrm{~mm})$ as shown in Fig. 11 along with their corresponding mesh profile. After serving for the same Gaussian pulses as input signals, the simulated waveforms at

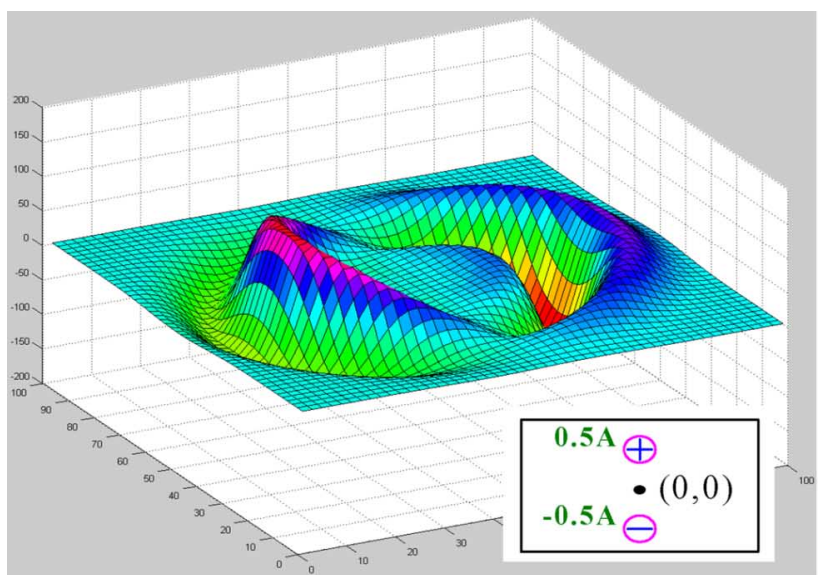

(a)

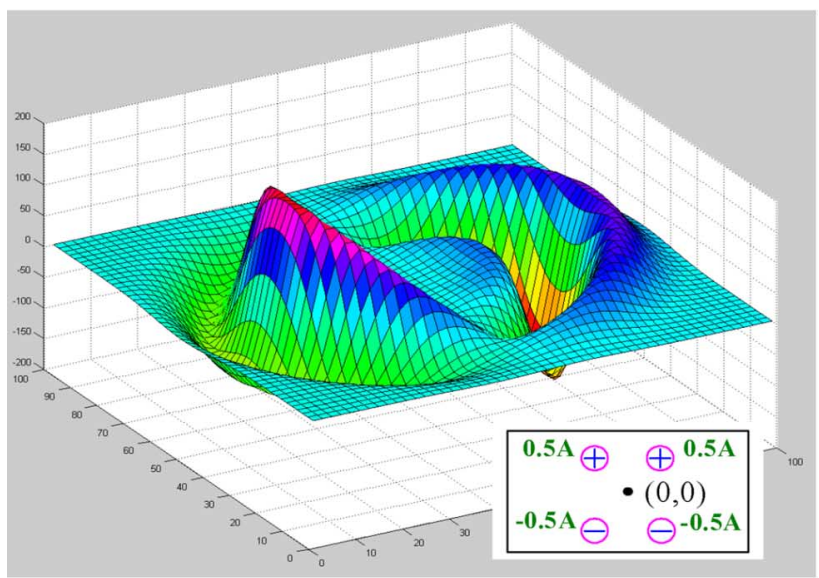

(b)

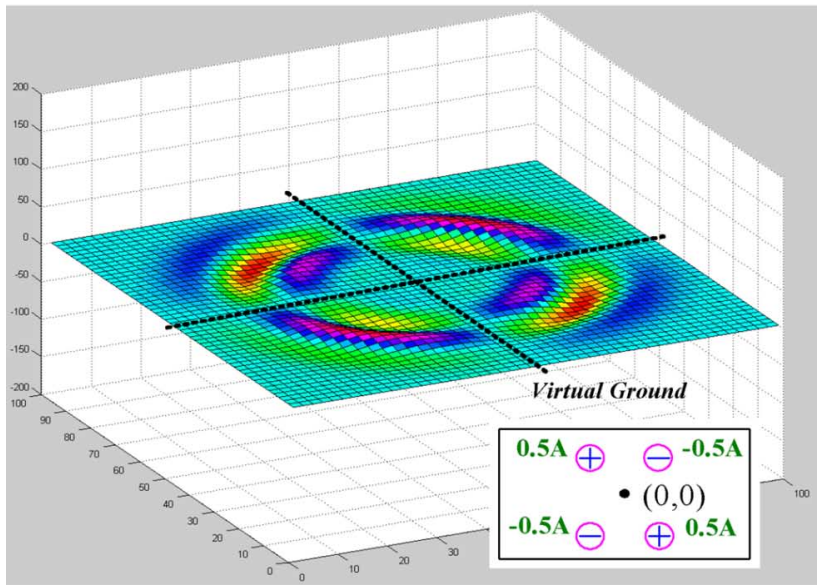

(c)

Fig. 13. At time of $400 \mathrm{ps}$, the overall electric-field patterns of three cases of differential-source settings in reference to Fig. 12. (a) Case 1: one pair of differential sources. (b) Case 2: two pairs of differential sources with the same polarity. (c) Case 3: two anti-polarity pairs of differential sources.

the probe are presented in Fig. 12 while three cases of source settings are investigated. Compared with the noise waveform of one pair of differential sources, the signal allocations of multiple differential-sources diversely influence the induced voltage noise.

For the more detailed understanding, Fig. 13 displays the overall electric-field patterns at the time of $400 \mathrm{ps}$ for three cases 


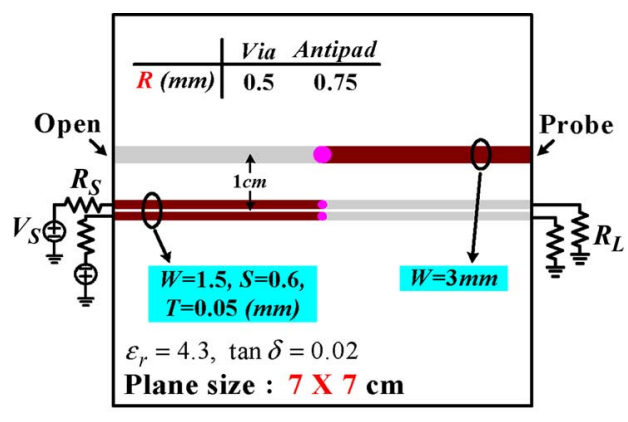

(a)

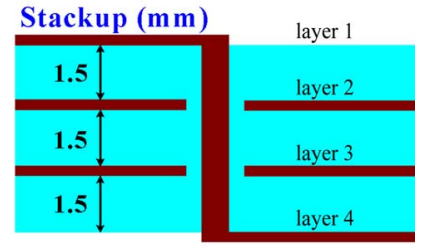

(b)

Fig. 14. Specifications and measurement settings of test board. (a) Top view. (b) Side view.

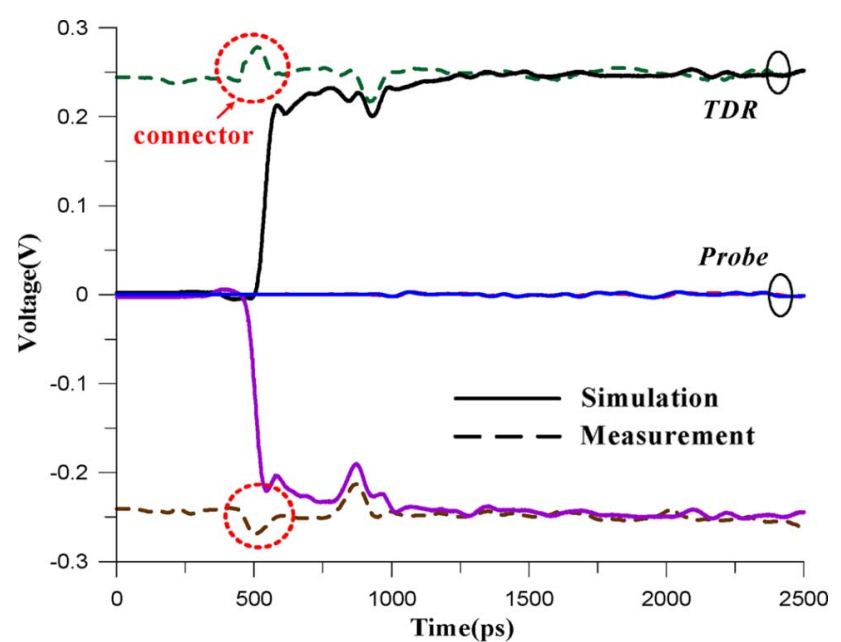

(a)

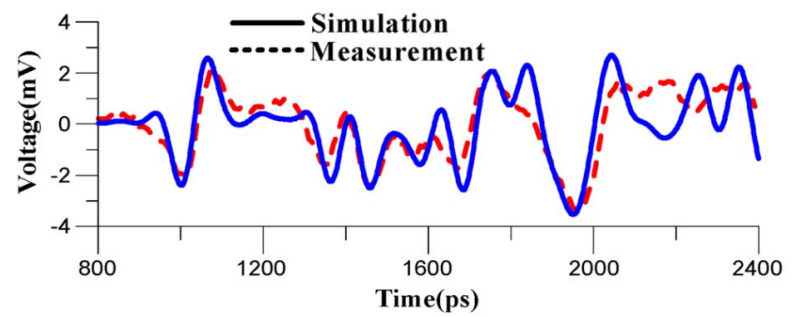

(b)

Fig. 15. Comparisons between the simulated and measured waveforms at both the TDR end and the probe as in Fig. 14. (a) The TDR waveforms. (b) The waveforms at the probe.

of differential-source settings on the plane. Note that the outward-traveling electric field of Case 3 (the differential-sources with antipolarity) is the smallest fluctuation since the appearance of two virtual grounds provided by the positive-and-negative polarity alternates the signal allocation.

\section{EXPERIMENTAL VERIFICATION}

In order to verify the accuracy of hybrid techniques, a test board was fabricated and measured by TEK/CSA8000B timedomain reflectometer. The designed test board comprises the single-ended and differential-via structures, connecting with the corresponding top- and bottom-layer traces. The design specifications and measurement settings of test board are illustrated in Fig. 14.

To perform the time-domain simulation, the launching voltage sources are drawn out of reflectometer. As the driving

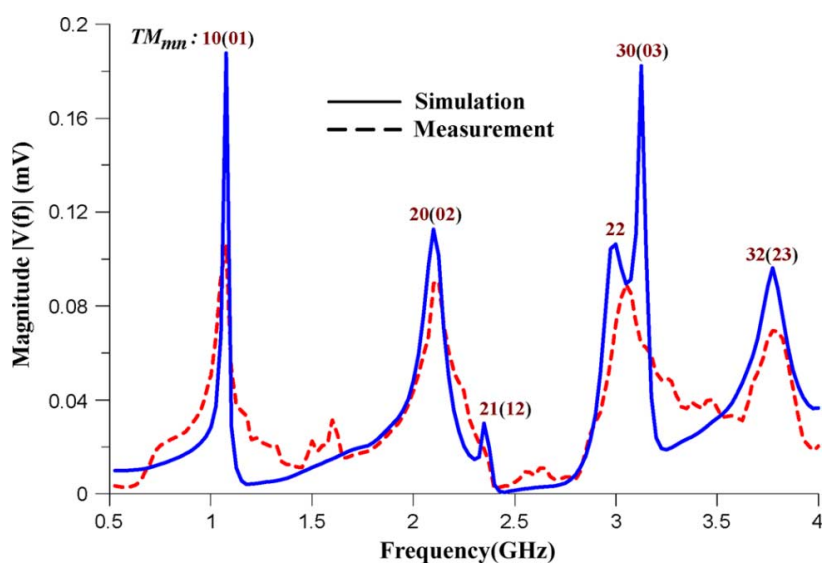

Fig. 16. Frequency-domain magnitude of the probing waveforms corresponding to Fig. 15(b) and the plane resonances.

signals pass through the differential vias, the parallel-plate structure is excited, incurring the SSN within the planes. Later, the quiet trace will suffer form this voltage noise through the single-ended via transition. After extracting the equivalent circuit models of coupled-via structures and well dividing the parallel plates, the SI/PI co-analysis for test board can be achieved. Simulation results are compared with the measurement data as shown in Fig. 15 accordingly.

As observed in Fig. 15(a), the differential signals have the internal skew of about $30 \mathrm{ps}$ and the bulgy noise arising at about $500 \mathrm{ps}$ is due to the series-wound connector used in the measurement. The capacitive effect of via discontinuities is occurred at about 900 ps, while the deviations between the simulation and measurement are attributed to the excessive high-frequency loss of input signals. For the zoom-in view of probing waveforms as in Fig. 15(b), it is displayed that the comparison is still in good agreement except for the lossy effect not included in the time-domain simulation. Applying the fast Fourier transform, the frequency-domain magnitude of probing waveforms is obtained in Fig. 16. In addition to the similar trend of time-domain simulation and measurement results, the peak frequencies correspond to the parallel-plate resonances of test board exactly. Hence, the exactitude of the proposed hybrid techniques can be verified.

\section{CONCLUSION}

A hybrid time-domain technique has been introduced and applied successfully to perform the SI/PI co-analysis for the differential-via transitions in the multilayer PCBs. The signal 
propagation on the differential traces is characterized by the known telegrapher's equations and the parallel-plate structure is discretized by the combined FETD/FDTD mesh schemes. The coarser FDTD segmentation for most of regular regions interfaces with an unconditionally stable FETD mesh for the local region having the differential-via transitions inside.

In use of hybrid techniques, the computational time and memory requirement are therefore far less than those of a traditional FDTD space with the finer mesh resolution but preserve the same degrees of numerical accuracy throughout the simulation.

In face of the assemblages of multiple signal transitions in the specific areas, the hybrid techniques still can be adopted by slightly modifying the mesh profiles in the local FETD regions. Furthermore, the numerical results demonstrate that the best signal allocation for PI consideration is positive-and-negative alternate. Once the boundary conditions between the FETD and FDTD regions are well defined, it is expected that the hybrid techniques have a great ability to deal with the more realistic problems of high-speed interconnect designs concerned in the signal traces touted through the multilayer structures.

\section{REFERENCES}

[1] K. S. Yee, "Numerical solution of initial boundary value problems involving Maxwell's equations in isotropic media," IEEE Trans. Antennas Propag., vol. AP-14, no. 3, pp. 302-307, May 1966.

[2] K. S. Kunz and R. J. Luebbers, The Finite Difference Time Domain Method for Electromagnetics. Boca Raton, FL: CRC, 1993, ch. 2, 3.

[3] S. V. Georgakopoulos, R. A. Renaut, C. A. Balanis, and C. R. Birtcher, "A hybrid fourth-order FDTD utilizing a second-order FDTD subgrid," IEEE Microw. Wireless Compon. Lett., vol. 11, no. 11, pp. 462-464, Nov. 2001.

[4] M. F. Hadi and M. Piket-May, "A modified $\operatorname{FDTD}(2,4)$ scheme for modeling electrically large structures with high-phase accuracy," IEEE Trans. Antennas Propag., vol. 45, no. 2, pp. 254-264, Feb. 1997.

[5] A. R. Bretones, R. Mittra, and R. G. Martin, "A hybrid technique combining the method of moments in the time domain and FDTD," IEEE Microw. Guided Wave Lett., vol. 8, no. 8, pp. 281-283, Aug. 1998.

[6] A. Monorchio, A. R. Bretones, R. Mittra, G. Manara, and R. G. Martin, "A hybrid time-domain technique that combines the finite element, finite difference and method of moment techniques to solve complex electromagnetic problems," IEEE Trans. Antennas Propag., vol. 52, no. 10, pp. 2666-2674, Oct. 2004.

[7] R.-B. Wu and T. Itoh, "Hybrid finite-difference time-domain modeling of curved surfaces using tetrahedral edge elements," IEEE Trans. Antennas Propag., vol. 45, no. 8, pp. 1302-1309, Aug. 1997.

[8] D. Koh, H.-B. Lee, and T. Itoh, "A hybrid full-wave analysis of viahole grounds using finite-difference and finite-element time-domain methods," IEEE Trans. Microw. Theory Tech., vol. 45, no. 12, pt. 2, pp. 2217-2223, Dec. 1997.

[9] S. Chun, J. Choi, S. Dalmia, W. Kim, and M. Swaminathan, "Capturing via effects in simultaneous switching noise simulation," in Proc. IEEE Int. Symp. Electromagn. Compat., Aug. 2001, vol. 2, pp. 1221-1226.

[10] J.-N. Hwang and T.-L. Wu, "Coupling of the ground bounce noise to the signal trace with via transition in partitioned power bus of PCB," in Proc. IEEE Int. Symp. Electromagn. Compat., Aug. 2002, vol. 2, pp. 733-736.

[11] J. Park, H. Kim, J. S. Pak, Y. Jeong, S. Baek, J. Kim, J. J. Lee, and J. J. Lee, "Noise coupling to signal trace and via from power/ground simultaneous switching noise in high speed double data rates memory module," in Proc. IEEE Int. Symp. Electromagn. Compat., Aug. 2004, vol. 2, pp. 592-597.

[12] S.-M. Lin and R.-B. Wu, "Composite effects of reflections and ground bounce for signal vias in multi-layer environment," in Proc. IEEE Microwave Conf. APMC, Dec. 2001, vol. 3, pp. 1127-1130.

[13] "Simulation Package for Electrical Evaluation and Design (SpeedXP)" Sigrity Inc., Santa Clara, CA [Online]. Available: www.sigrity.com
[14] “High Frequency Structure Simulator” ver. 9.1, Ansoft Co., Pittsburgh, PA [Online]. Available: www.ansoft.com

[15] J. Jin, The Finite Element Method in Electromagnetics. New York: Wiley, 1993, ch. 12.

[16] N. M. Newmark, "A method of computation for structural dynamics," J. Eng. Mech. Div., ASCE, vol. 85, pp. 67-94, Jul. 1959.

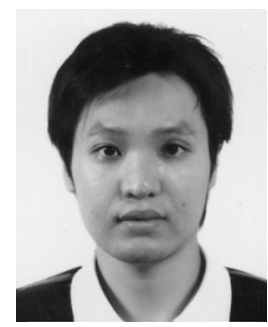

Wei-Da Guo was born in Taoyuan, Taiwan, R.O.C., on September 25, 1981. He received the B.S. degree in communication engineering from Chiao-Tung University, Hsinchu, Taiwan, R.O.C., in 2003, and is currently working toward the Ph.D. degree in communication engineering at National Taiwan University, Taipei, Taiwan, R.O.C.

His research topics include computational electromagnetics, SI/PI issues in the design of high-speed digital systems.

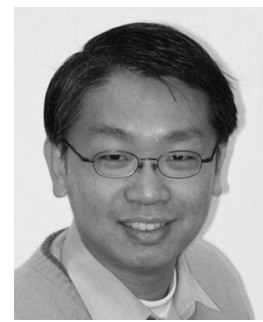

Guang-Hwa Shiue was born in Tainan, Taiwan, R.O.C., in 1969. He received the B.S. and M.S. degrees in electrical engineering from National Taiwan University of Science and Technology, Taipei, Taiwan, R.O.C., in 1995 and 1997, respectively, and the Ph.D. degree in communication engineering from National Taiwan University, Taipei, in 2006.

$\mathrm{He}$ is a Teacher in the Electronics Department of Jin-Wen Institute of Technology, Taipei, Taiwan. His areas of interest include numerical techniques in electromagnetics, microwave planar circuits, signal/power integrity (SI/PI) and electromagnetic interference (EMI) for high-speed digital systems, and electrical characterization of system-in-package.

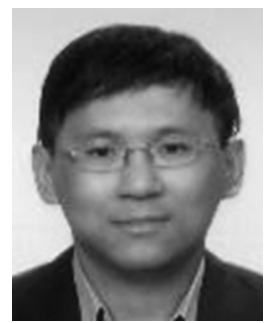

Chien-Min Lin (M'92) received the B.S. degree in physics from National Tsing Hua University, Hsinchu, Taiwan, R.O.C., the M.S. degree in electrical engineering from National Taiwan University, Taipei, Taiwan, R.O.C., and the Ph.D. degree in electrical engineering from the University of Washington, Seattle.

He was with IBM, where he worked on the xSeries server development and Intel, where he worked on advanced platform design. In January 2004, he joined Taiwan Semiconductor Manufacturing Company, Ltd., Taiwan, as a Technical Manager in packaging design and assembly validation. He has been working on computational electromagnetics for the designs of microwave device and rough surface scattering, signal integrity analysis for high-speed interconnect, and electrical characterization of system-in-package.

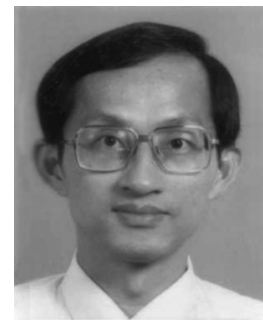

Ruey-Beei Wu (M'91-SM'97) received the B.S.E.E. and $\mathrm{Ph} . \mathrm{D}$. degrees from National Taiwan University, Taipei, Taiwan, R.O.C., in 1979 and 1985, respectively.

In 1982, he joined the faculty of the Department of Electrical Engineering, National Taiwan University, where he is currently a Professor and the Department Chair. He is also with the Graduate Institute of Communications Engineering established in 1997. From March 1986 to February 1987, he was a Visiting Scholar at the IBM East Fishkill Facility, NY. From August 1994 to July 1995, he was with the Electrical Engineering Department, University of California at Los Angeles. He was also appointed Director of the National Center for High-Performance Computing (1998-2000) and has served as Director of Planning and Evaluation Division since November 2002, both under the National Science Council. His areas of interest include computational electromagnetics, microwave and millimeter-wave planar circuits, transmission line and waveguide discontinuities, and interconnection modeling for computer packaging. 